\title{
Device-based Probe of Triplet Exciton Diffusion in Singlet Fission Materials
}

Tao Zhang ${ }^{\dagger}$, Deepesh Rai ${ }^{\dagger}$ and Russell J. Holmes

Department of Chemical Engineering and Materials Science, University of Minnesota,

Minneapolis, MN 55455, United States

${ }^{\dagger}$ Equal contribution to this work

Supporting Information 
(a)

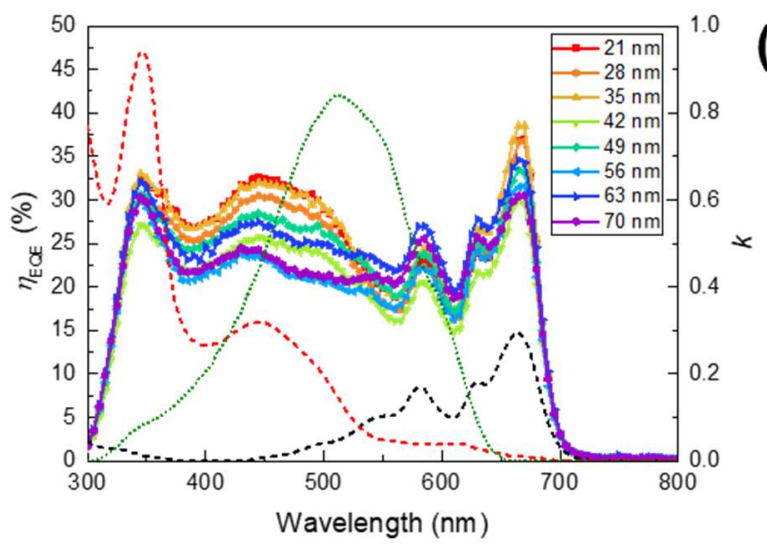

(b)

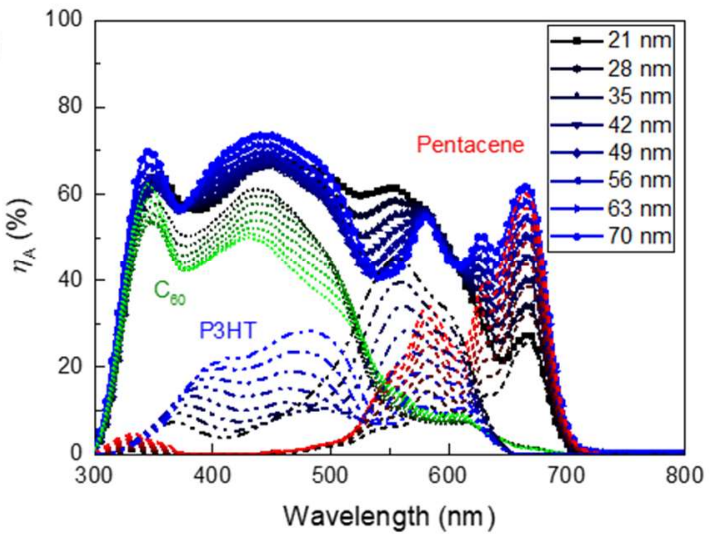

(c)

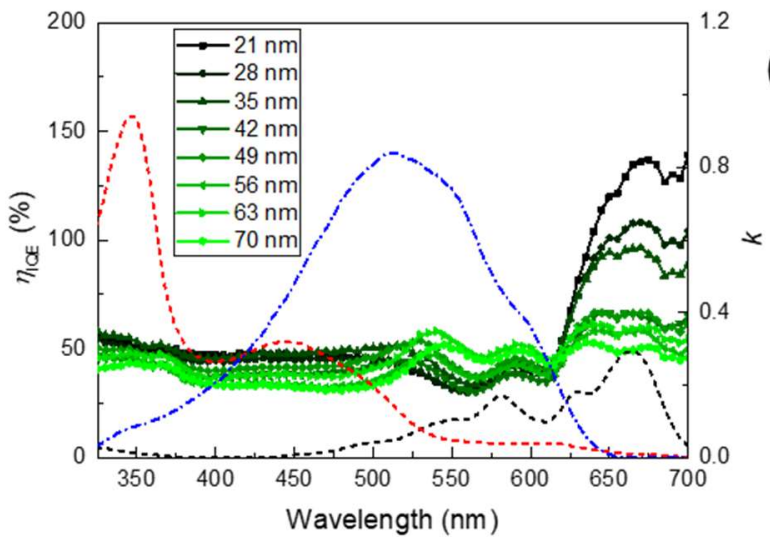

(d)

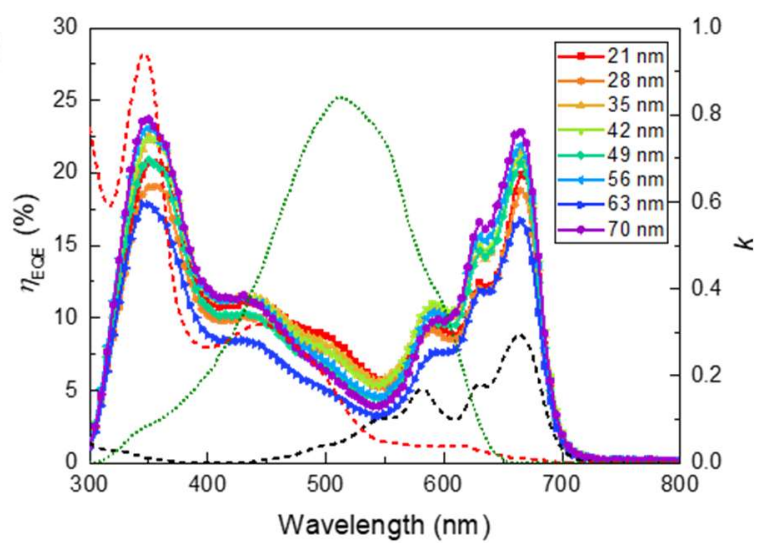

(e)

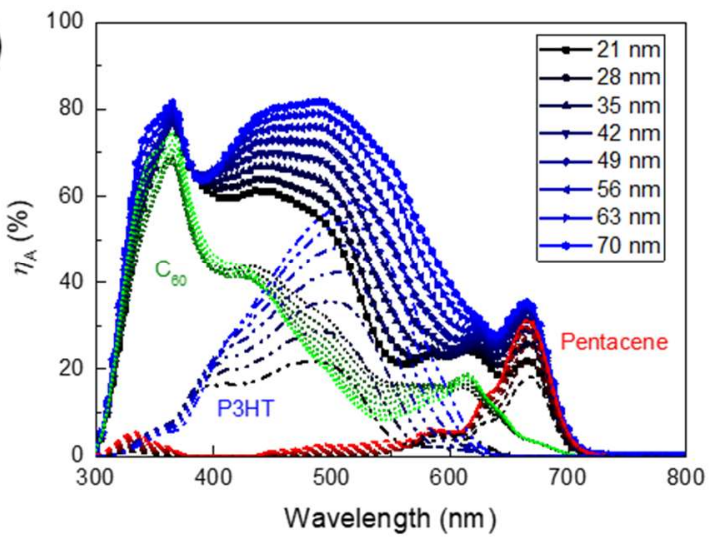

(f)

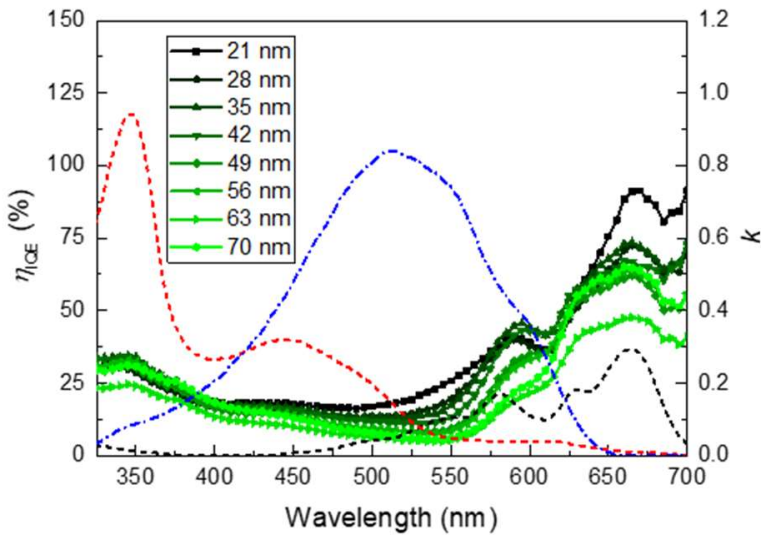

Figure S1 (a) External quantum efficiency spectra for pentacene- $\mathrm{C}_{60}$ planar photovoltaic cells. The $\eta_{E Q E}$ spectra are measured at short-circuit as a function of pentacene layer thickness. Devices have the structure: ITO/20 nm P3HT/X (=21-70) nm pentacene $/ 45 \mathrm{~nm} \mathrm{C}{ }_{60} / 10 \mathrm{~nm} \mathrm{BCP} / 1 \mathrm{~nm} \mathrm{MoO} / 100$ $\mathrm{nm} \mathrm{Al}$. (b) $\eta_{A}$ spectra for the devices in (a) calculated using an optical transfer matrix model. (c) Corresponding $\eta_{I Q E}$ spectra calculated by dividing the $\eta_{E Q E}$ spectra in (a) by the $\eta_{A}$ spectra in (b). (d) $\eta_{E Q E}$ spectra as a function of pentacene layer thickness for devices having the structure: ITO/20 $\mathrm{nm}$ P3HT/X (=21-70) nm pentacene $/ 75 \mathrm{~nm} \mathrm{C} 60 / 10 \mathrm{~nm} \mathrm{BCP} / 1 \mathrm{~nm} \mathrm{MoO}_{\mathrm{x}} / 100 \mathrm{~nm} \mathrm{Al}$. (e) $\eta_{A}$ spectra for the devices in (d). (f) $\eta_{I Q E}$ spectra calculated by dividing the $\eta_{E Q E}$ spectra in (d) by the $\eta_{A}$ spectra in (e). The extinction coefficients $(k)$ of pentacene (black line), $\mathrm{C}_{60}$ (red line), and P3HT (blue line) are also shown. 


\section{(a)}
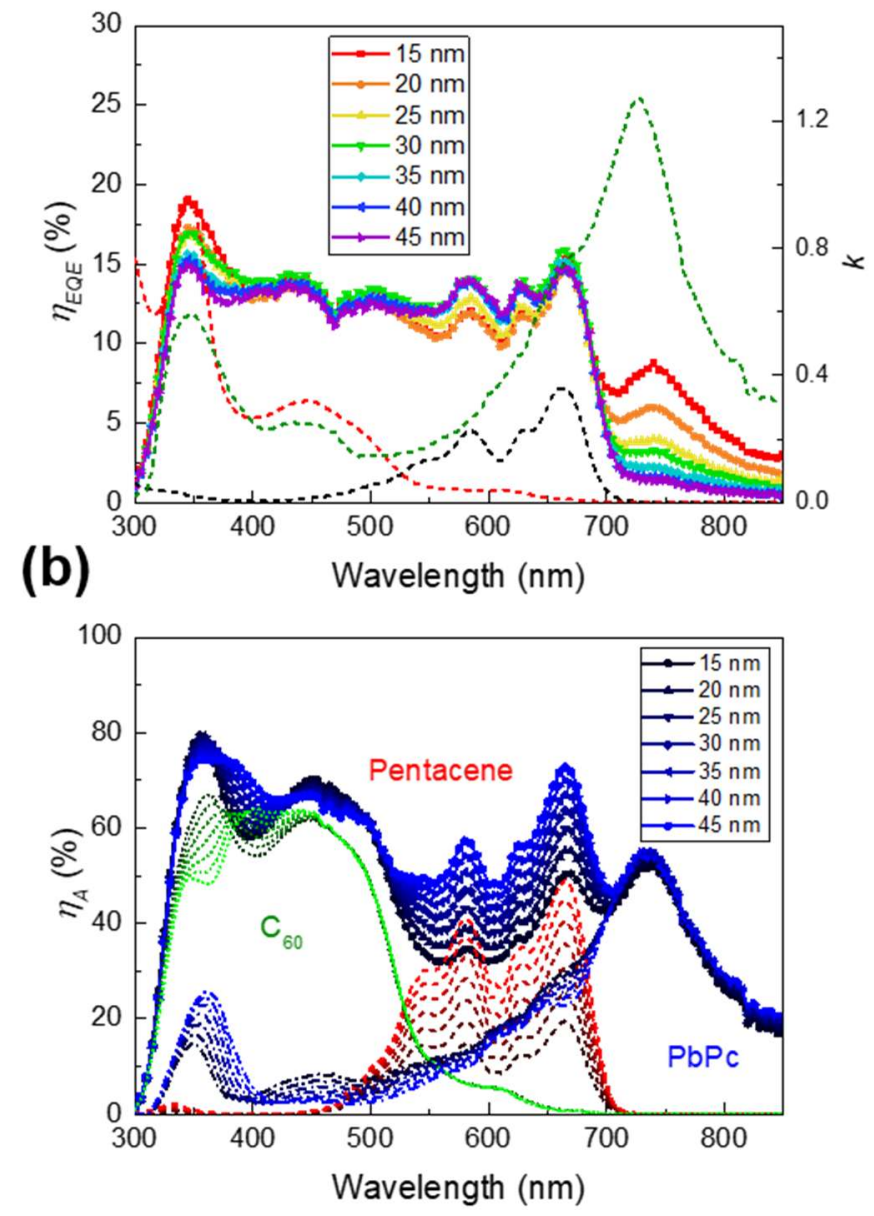

Figure S2 (a) External quantum efficiency spectra for $\mathrm{PbPc}$-pentacene- $\mathrm{C}_{60}$ planar photovoltaic cells. Spectra measured at short-circuit as a function of pentacene layer thickness for devices with the structure: ITO/10 nm TAPC/10 nm PbPc/X (=15-45) nm pentacene/35 nm C $60 / 10 \mathrm{~nm} \mathrm{BCP/1}$ $\mathrm{nm} \mathrm{MoO}_{\mathrm{x}} / 100 \mathrm{~nm} \mathrm{Al}$. The extinction coefficients $(k)$ of pentacene (black line), $\mathrm{C}_{60}$ (red line), and $\mathrm{PbPc}$ (green line) are also shown. (b) $\eta_{A}$ spectra for the devices in (a) calculated using an optical transfer matrix model. 
(a)

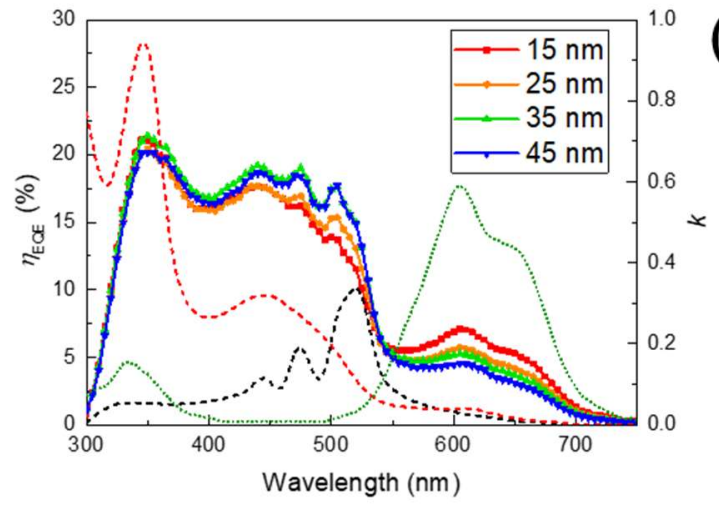

(b)

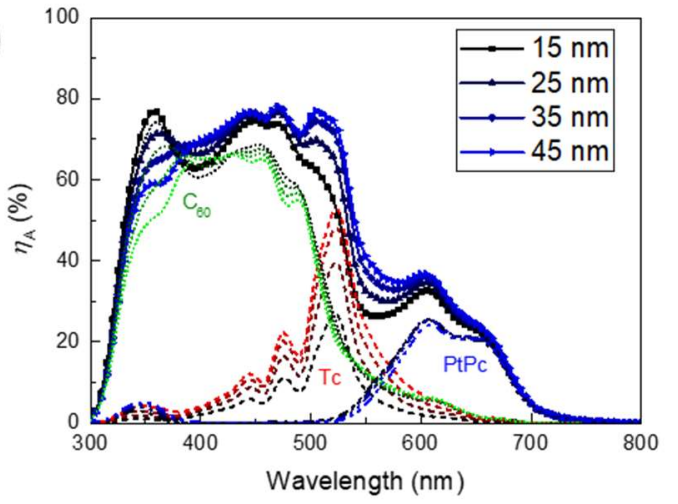

(c)

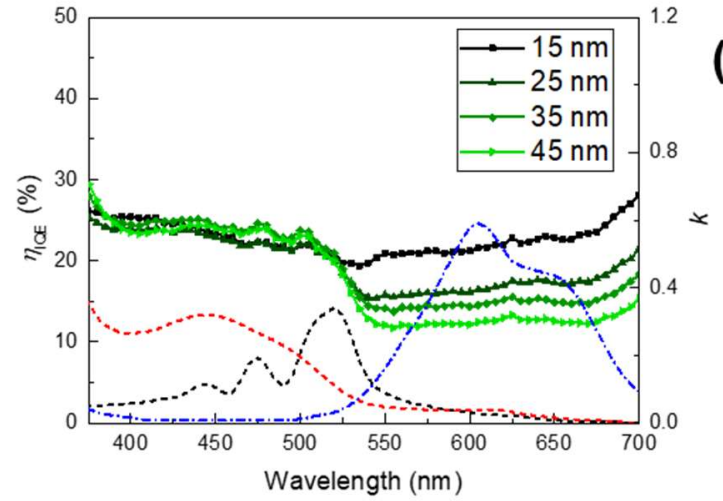

(d)

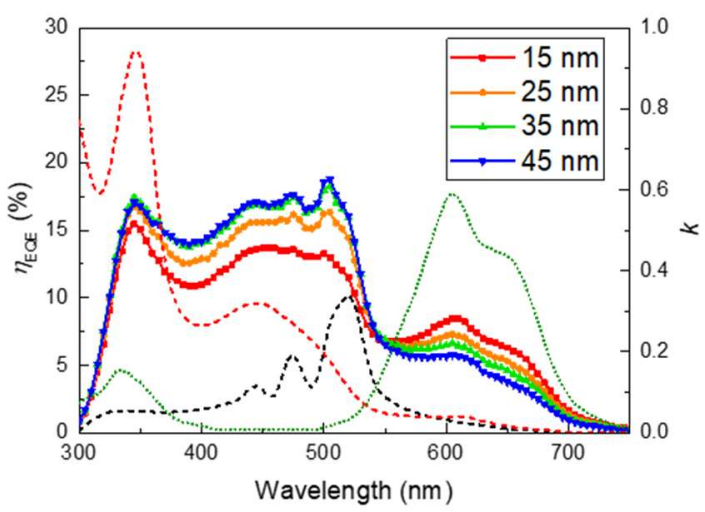

(e)

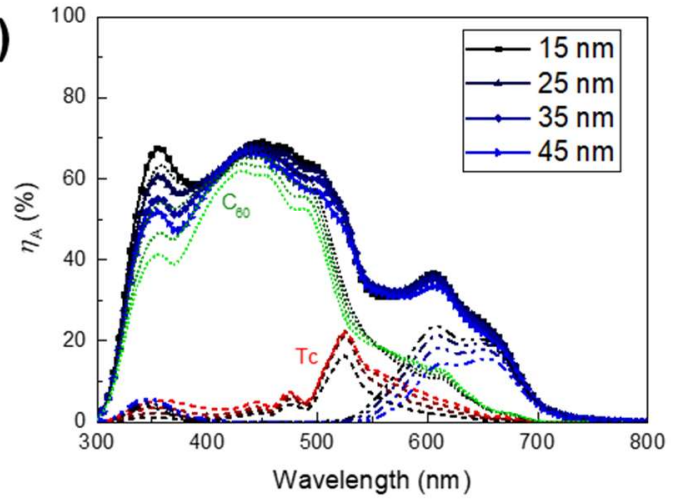

(f)

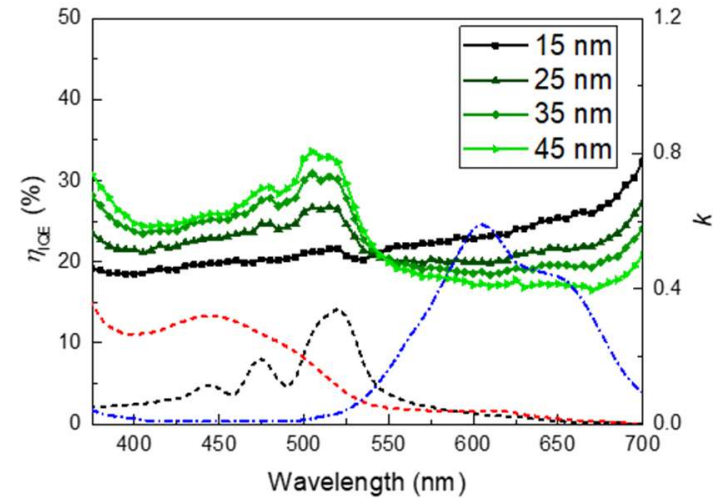

Figure S3 (a) External quantum efficiency spectra for PtPc-tetracene- $\mathrm{C}_{60}$ planar photovoltaic cells. The $\eta_{E Q E}$ spectra are measured at short-circuit as a function of tetracene layer thickness. Devices have the structure: ITO/10 nm TAPC/10 nm PtPc/X (=15-45) nm tetracene $/ 35 \mathrm{~nm} \mathrm{C} \mathrm{C}_{60} / 10 \mathrm{~nm}$ $\mathrm{BCP} / 1 \mathrm{~nm} \mathrm{MoO}_{\mathrm{x}} / 100 \mathrm{~nm}$ Al. (b) $\eta_{A}$ spectra for the devices in (a) calculated using an optical transfer matrix model. (c) Corresponding $\eta_{I Q E}$ spectra calculated by dividing the $\eta_{E Q E}$ spectra in (a) by the $\eta_{A}$ spectra in (b). (d) $\eta_{E Q E}$ spectra as a function of tetracene layer thickness for devices having the structure: ITO/10 nm TAPC/10 nm PtPc/X (=15-45) nm tetracene $/ 50 \mathrm{~nm} \mathrm{C} \mathrm{C}_{60} / 10 \mathrm{~nm}$ $\mathrm{BCP} / 1 \mathrm{~nm} \mathrm{MoO} / 100 \mathrm{~nm}$ Al. (e) $\eta_{A}$ spectra of devices in (d). (f) $\eta_{I Q E}$ spectra calculated by dividing the $\eta_{E Q E}$ spectra in (d) by the $\eta_{A}$ spectra in (e). The extinction coefficients $(k)$ of tetracene (black line), $\mathrm{C}_{60}$ (red line), and PtPc (blue line) are also shown. 

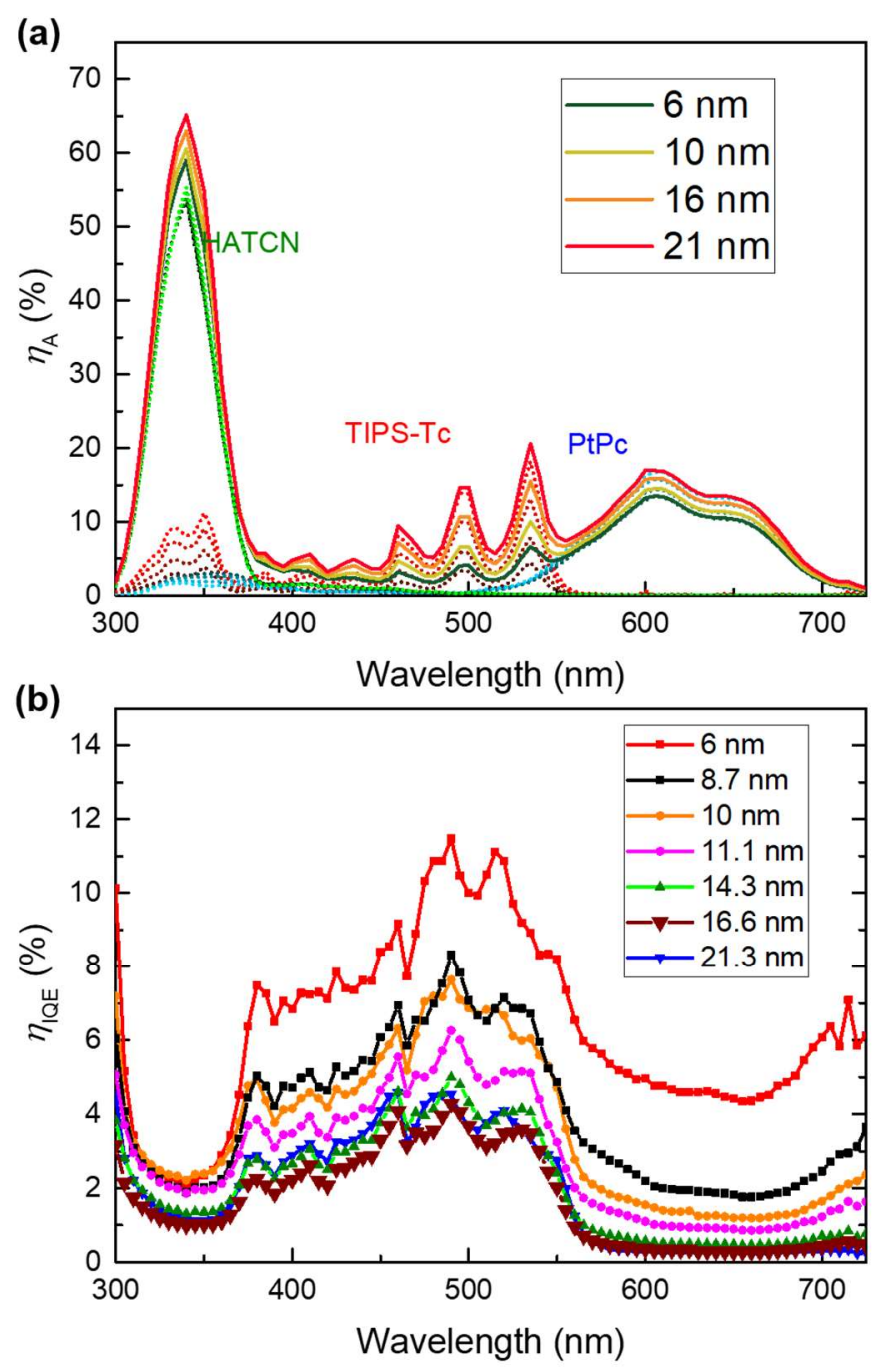

Figure S4 (a) The $\eta_{A}$ spectra of active layer calculated using transfer matrix model. (b) The $\eta_{I Q E}$ spectra calculated by dividing the $\eta_{E Q E}$ spectra in Fig. $5 \mathrm{~b}$ with the $\eta_{A}$ spectra in (a). 
(a)
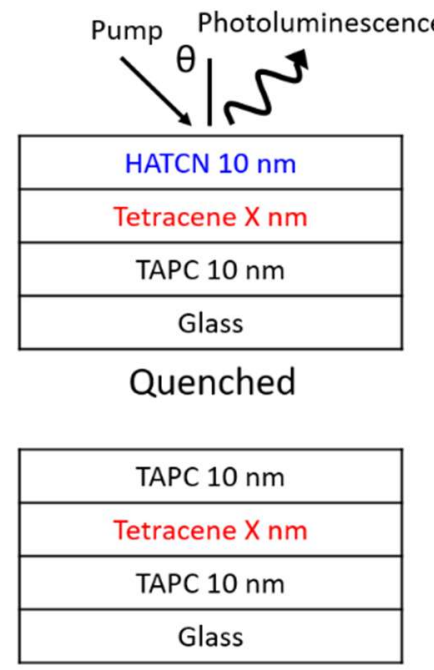

Unquenched (b)

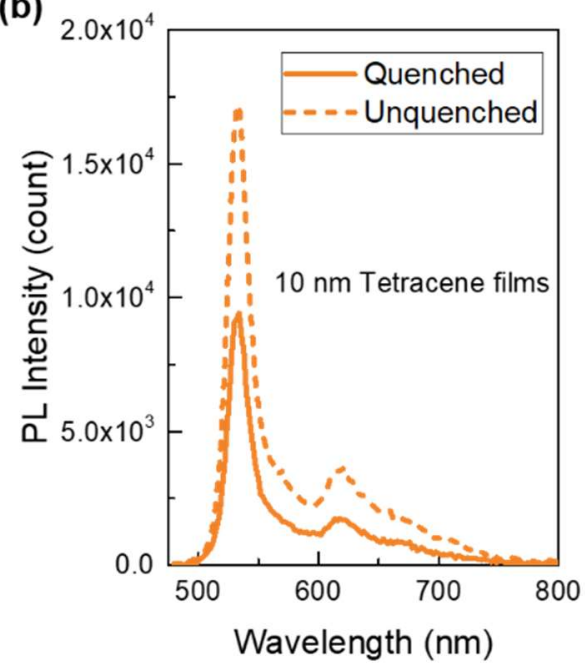

(c)

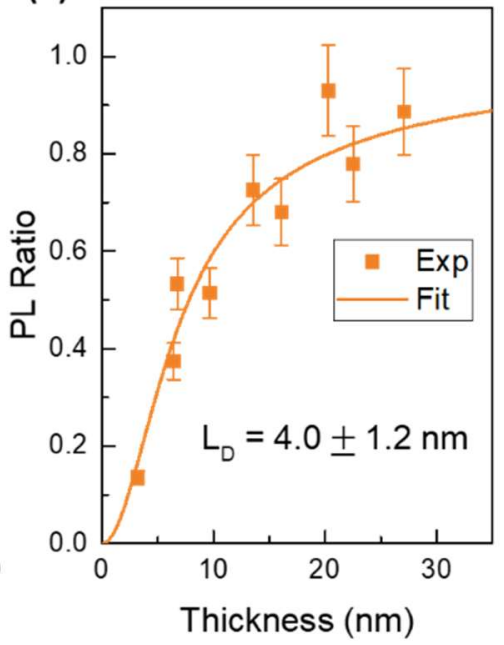

Figure S5 (a) Architectures for tetracene singlet $\mathrm{L}_{D}$ measurements. Films are all pumped with light having a wavelength of $\lambda=450 \mathrm{~nm}$ at an incident angle of $70^{\circ}$. (b) Representative PL spectra (10nm-thick tetracene) for the samples in (a). (c) The PL ratio of tetracene films as a function of tetracene thickness. 
(a)

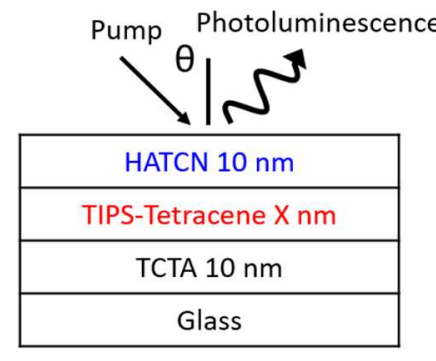

Quenched

\begin{tabular}{|c|}
\hline TAPC $10 \mathrm{~nm}$ \\
\hline TIPS-Tetracene $X \mathrm{~nm}$ \\
\hline TCTA $10 \mathrm{~nm}$ \\
\hline Glass \\
\hline
\end{tabular}

Unquenched (b)

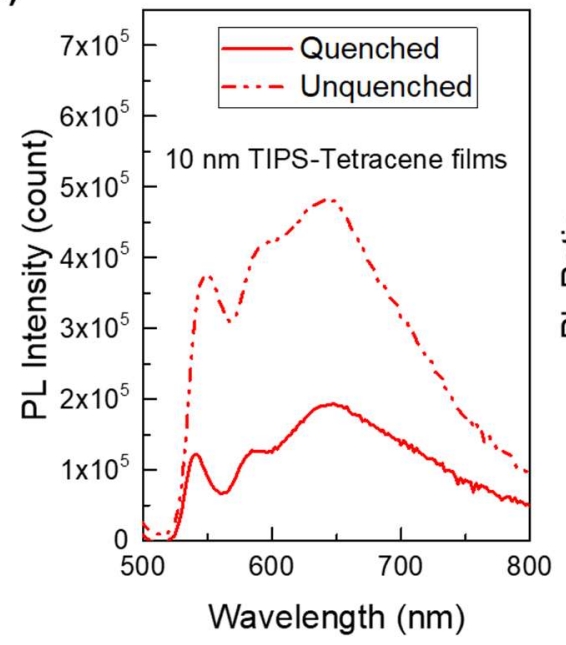

(c)

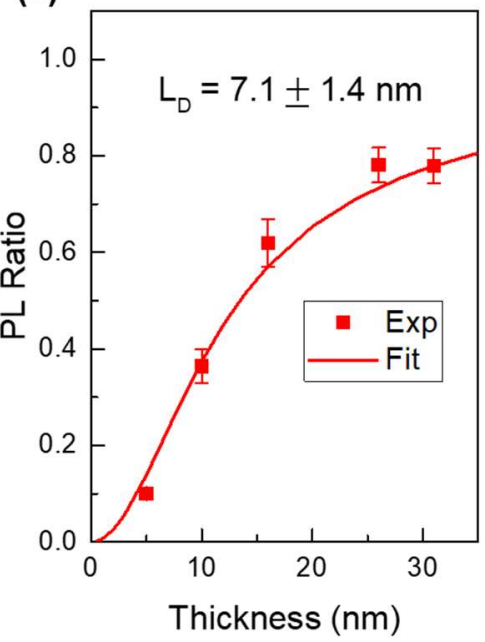

Figure S6 (a) Architectures for the measurement of TIPS-tetracene singlet $\mathrm{L}_{\mathrm{D}}$ by thicknessdependent photoluminescence (PL) quenching. Films are pumped with light having a wavelength of $\lambda=470 \mathrm{~nm}$ at an incident angle of $70^{\circ}$. (b) Representative PL spectra (10-nm-thick TIPStetracene) for the samples in (a). (c) The PL ratio of TIPS-tetracene films as a function of TIPStetracene thickness. 